\title{
Study of Feynman Scaling in Very Forward Neutron and Photon Production in Deep-Inelastic Scattering at HERA
}

\author{
Hamlet ZOHRABYAN* (Deutsches Elektronen-Synchrotron ( DESY)) \\ E-mail: hamlet.zohrabian@desy.de
}

\begin{abstract}
Measurements of very forward neutron and photon production in deep-inelastic $e p$ scattering at HERA as a function of Feynman- $x$ are presented.

The data are taken with the H1 detector in the years 2006 and 2007 and correspond to an integrated luminosity of $126 \mathrm{pb}^{-1}$. The analysis covers the range of negative four momentum transfer squared at the positron vertex $6<Q^{2}<100 \mathrm{GeV}^{2}$, inelasticity $0.05<y<0.6$ and the centerof-mass energy of the virtual photon-proton system $70<W<250 \mathrm{GeV}$. The dependence of the cross sections on $W$ is investigated. Predictions from DIS models and from models of the hadronic interactions of high energy Cosmic Rays are compared with the measurements.
\end{abstract}

XXI International Workshop on Deep-Inelastic Scattering and Related Subjects 22-26 April, 2013

Marseilles, France

${ }^{*}$ Speaker. 


\section{Introduction}

Measurements of particle production at very small angles with respect to the proton beam direction (forward direction) in $e p$ collision are important for the understanding of proton fragmentation. Measurements of forward particle also provide important constraints for modeling of high energy air showers and thereby are very valuable for the understanding of high energy cosmic ray data.

New results from the H1 Experiment on very forward photon and neutron production in deepinelastic positron-proton scattering (DIS) are reported [1]. The photons and neutrons are measured in the Forward Neutron Calorimeter (FNC) [2,3], which was installed at $106 \mathrm{~m}$ from the $e p$ interaction point at a polar angel $0^{\circ}$ with respect to the proton beam direction at the interaction point. The acceptance of the FNC is defined by the aperture of the HERA beam-line magnets and is limited to scatering angles of $\theta<0.8 \mathrm{mrad}$.

\section{Forward Photon and Neutron Spectra in DIS}

Differential cross sections normalised to the inclusive DIS cross sections are measured for forward photons and neutrons as a function of the Feynman- $x$ variable $x_{F}=p_{\|}^{*} / p_{\| \max }^{*}$ for three $W$ ranges: $70<W 1<130 \mathrm{GeV}, 130<W 2<190 \mathrm{GeV}$ and $190<W 3<250 \mathrm{GeV}$. The measurements are shown in Figures 1 and 2 together with the Monte Carlo (MC) model predictions.
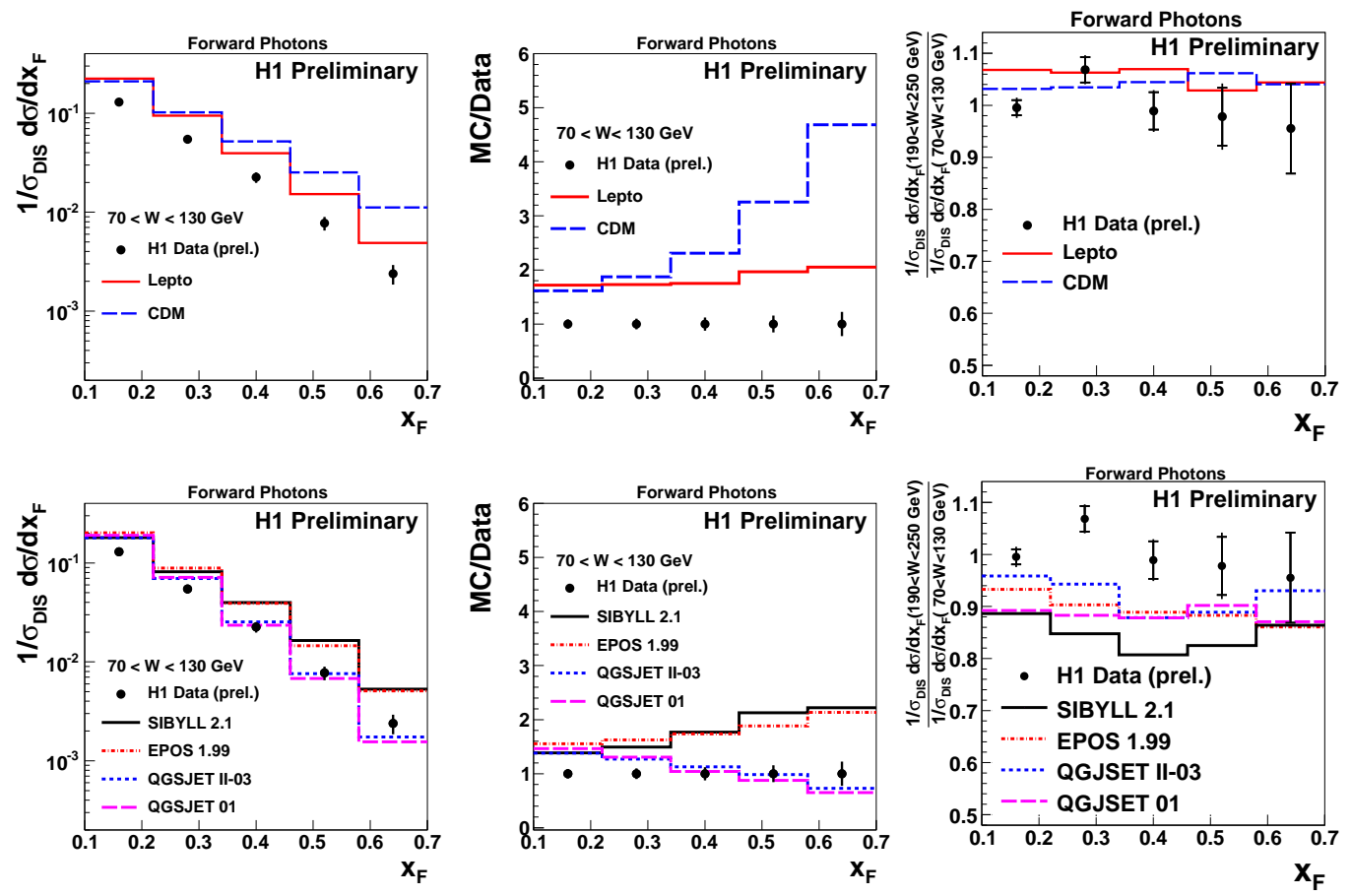

Figure 1: Normalized cross sections for forward photon production as a function of $x_{F}$ compared to predictions of the LEPTO and CDM MC models (upper row), and Cosmic Ray hadronic interaction models (lower row). The left column shows the $x_{F}$ distributions, the middle column shows the ratios of MC predictions to the data, and the right column shows the ratio of $x_{F}$ spectra in the $W$ range $190<W<250 \mathrm{GeV}$ to that in the $W$ range $70<W<130 \mathrm{GeV}$. 
The DJANGOH program [4] is used to generate inclusive DIS events. Higher order QCD effects are simulated using leading log parton showers as implemented in LEPTO [5], or using the Color Dipole Model (CDM) as implemented in ARIADNE [6]. In addition the RAPGAP [7] pionexchange model is used to describe the high $x_{F}$ part of forward neutrons spectra. The data are also compared with Cosmic Ray (CR) hadronic interaction models commonly used for the simulation of CR air shower cascades: EPOS [8], QGSJET 01 [9] [10], QGSJET II [11] [12] and SIBYLL [13] [14].

The left column of Figure 1 shows the cross sections measured for the most energetic photons with pseudorapidity $\eta>7.9$ as a function of Feynman- $x$ variable for $W$ region $(70-130 \mathrm{GeV})$ compared to DJANGOH and CR models. The distributions for the other $W$ ranges look very similar to this $W$ range. Both MC models, LEPTO and CDM, significantly overestimate the rate of forward photons by $\sim 70 \%$. The shape of the LEPTO prediction is close to the data, while CDM exhibits a harder $x_{F}$ behavior. The CR models are closer to data in absolute values, but are also higher by $\sim 30-50 \%$. The best description of the forward photon measurements is provided by QGSJET model. The right side of Figure 1 shows the ratio of the $x_{F}$ spectrum in the $W$ range $190<W<250 \mathrm{GeV}$ to that in the $W$ range $70<W<130 \mathrm{GeV}$. The ratio for the data is consistent with unity within errors, supporting the validity of Feynman scaling. The ratios for standard DIS models (LEPTO and CDM) are slightly larger, and the ratios for CR models are lower than unity.
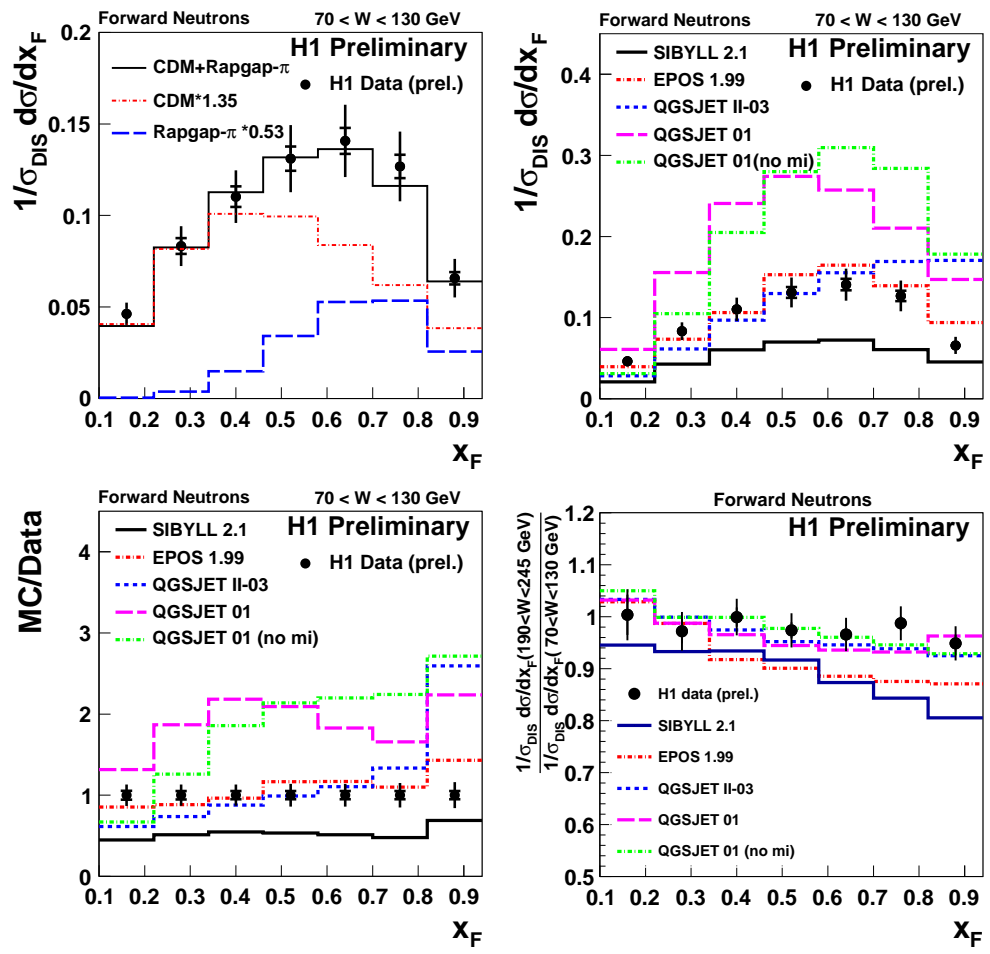

Figure 2: Normalized cross sections for forward neutron production as a function of $x_{F}$ compared to CDM, the RAPGAP pion-exchange model and their combination using weighting factors (upper left figure) and $\mathrm{CR}$ models (upper-right figure). The ratio of MC predictions to the measurement is shown in the lower left plot. The lower right plot shows the ratio of $x_{F}$ spectra in the $W$ range $190<W<245 \mathrm{GeV}$ to that in the range $70<W<130 \mathrm{GeV}$.

Figure 2 shows the results for the $x_{F}$ measurements of forward neutron production. In the 
upper left plot the data is compared to the standard CDM and RAPGAP- $\pi$ (pion-exchange) model and to the combination of these models. The 'combined' model describes the data well. The measured distribution is also compared to the CR interaction models in upper right panel of Figure 2. EPOS gives the best description of the data. The lower right plot shows the ratio of the $x_{F}$ spectrum in the $W$ range $190<W<245 \mathrm{GeV}$ to that in the range $70<W<130 \mathrm{GeV}$. This ratio for the data is consistent with being independent of $x_{F}$ within errors, while some CR models depend significantly on $x_{F}$ and therefore are inconsistent with Feynman scaling.

\section{Fraction of DIS events with forward photons and neutrons}

The measurement of forward photons and neutrons allows a test of the limiting fragmentation hypothesis, which implies that forward particle production is insensitive to $W$. To investigate this prediction, the ratio of the forward photon and neutron production cross section to the inclusive DIS cross section is measured as a function of $W$ and compared with the standard DIS and the CR models in Figure 3. Within the uncertainties the fraction of DIS events with photons and neutrons
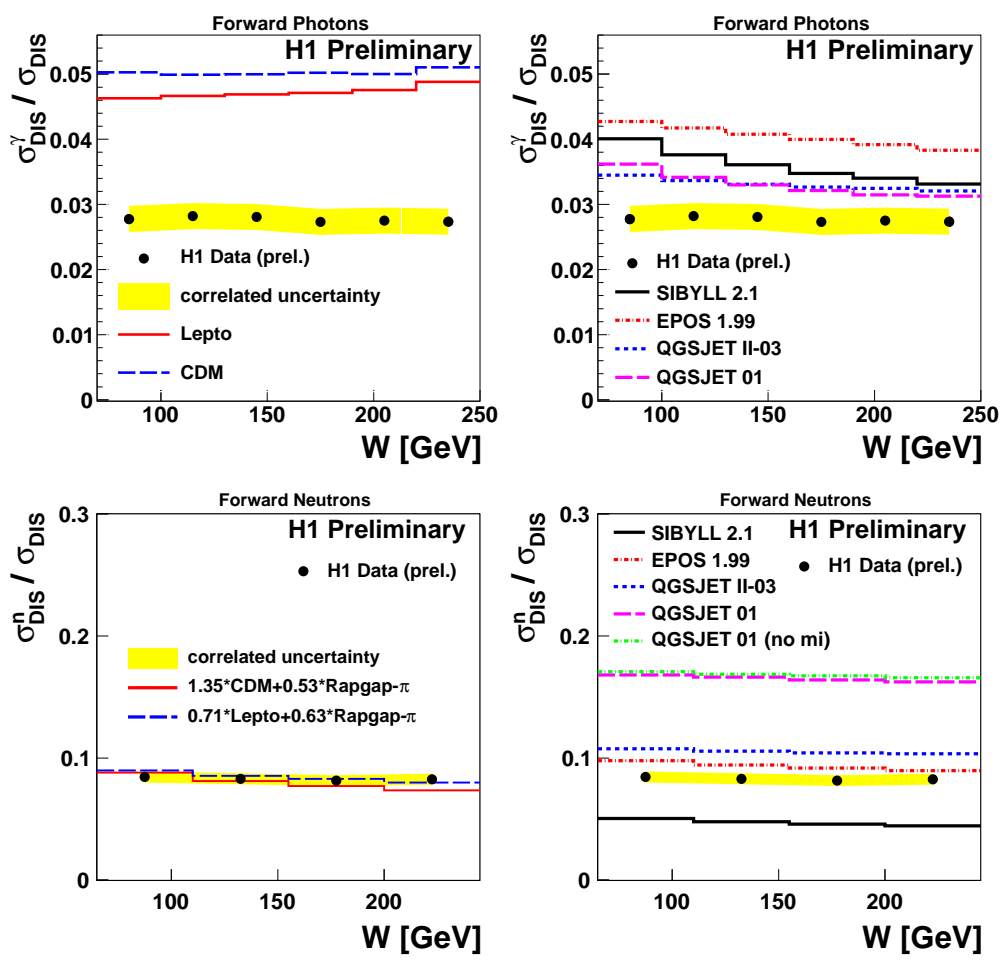

Figure 3: Fraction of DIS events with forward photons and neutrons as a function of $W$. Upper (lower) row shows the distributions for the forward photons(neutrons). The MC models are compared to the measurements.

is independent from $W$, in agreement with the hypothesis of limiting fragmentation. LEPTO and CDM indicate a significant differences in normalization compared to data as well as slight dependence of $W$. The CR models indicate a large spread of their predictions and some $W$ dependencies for both, the photon and the neutron yields. For forward neutrons the combination of the standard DIS and pion exchange models describe the ratio well. 


\section{Summary}

The production of forward photons and neutrons has been studied in deep inelastic positron-proton scattering in the kinematic range $6<Q^{2}<100 \mathrm{GeV}^{2}, 0.05<y<0.6$ and the pseudorapidity range of photons and neutrons $\eta>7.9$ in laboratory frame. The Monte Carlo models overestimate the rate of photons. LEPTO describes the shapes well, while CDM predicts a harder $x_{F}$ spectrum for $x_{F}$. The CR models considered are closer to the data in normalisation. For forward neutrons none of the models describes the data well. The combination of standard fragmentation and the pion-exchange models describes $x_{F}$ spectra well. The measurements support the limiting fragmentation hypothesis and are consistent with Feynman scaling. The present measurements provides further insight into the proton fragmentation process and can be used for models tuning.

\section{References}

[1] Forward photon spectra measured in FNC, H1prelim-13-012, http://www-h1.desy.de/psfiles/confpap/DIS2013/H1prelim-13-012.pdf

[2] F.D.Aaron et al. Measurement of Photon Production in the Very Forward Direction in Deep-Inelastic Scattering at HERA, Eur.Phys.J. C71 (2011) 1771

[3] F.D.Aaron et al. Measurement of Leading Neutron Production in Deep-Inelastic Scattering at HERA, Eur.Phys.J. C68 (2010) 381-399

[4] K.Charchula, G.A.Schuler and H.Spiesberger, DJANGOH 1.4, Comput.Phys.Commun. 81,381 (1994).

[5] G.Ingelman, A.Edin and J.Rathsman, LEPTO 6.5, Comput.Phys.Commun.101 (1997) 108 [hep-ph/9605286].

[6] L.Lonnblad, ARIADNE 4.10, Comput.Phys.Commun.71 (1992) 15.

[7] H. Jung, RAPGAP 3.1, Comp. Phys. Commun. 86 (1995) 147.

[8] K.Werner,F.-M.Liu and T.Pierog, Phys.Rev. C74 (2006) 044902 [hep-ph/0506232].

[9] N.N.Kalmykov and S.S.Ostapchenko, Phys.Atom.Nucl. 56 (1993) 346.

[10] N.N.Kalmykov, S.S.Ostapchenko, A.I.Pavlov, Nucl.Phys.Proc.Suppl.52B (1997) 17.

[11] S.S.Ostapchenko, Phys.Rev. D74 (2006) 014026 [hep-ph/0505259].

[12] S.S.Ostapchenko, AIP Conf.Proc.928 (2007) 118 [arXiv:0706.3784].

[13] J.Engel et al., Phys.Rev. D46 (1992) 5013.

[14] E.-J.Ahn et al., Phys.Rev. D80(2009)094003 [arXiv:0906.4113]. 